\title{
Analysis of Stem Tip Pain in Revision Total Knee Arthroplasty
}

\section{Abstract}

Stem tip pain following revision total knee arthroplasty is a significant cause of patient dissatisfaction, which in the presence of an aseptic well-fixed component has no widely accepted surgical solution. A definitive cause of stem tip pain remains elusive however it has been suggested that high stress concentrations within the region of the stem tip may play a role.. This paper reports a finite element study of a novel clinical technique where a plate is attached to the tibia within the region of the stem tip to reduce stem tip pain. The results demonstrate that the plate reduces stress concentrations in the bone at the stem tip of the implant. The magnitude of stress reduction is dependent upon plate location, material and attachment method.

\section{Introduction}

In revision Total Knee Arthroplasty (TKA) the operating surgeon may encounter difficulties in maintaining component alignment due to proximal tibial bone defects as a result of the primary TKA. In order to overcome the stability problems associated with these defects a tibial stem extension component is often used. However, one issue which has been reported with this method of fixation and has been identified previously in revision Total Hip Arthroplasty (THA) [1], is stem-tip pain. In a clinical study carried out by Barrack [2] a review of 50 TKA patients with a press-fitted stem extension over a 2 year minimum followup period identified pain in the stem tip of the tibial component in $14 \%$ of the patients. For the 16 patients reviewed with cemented tibial stems the clinical study saw an occurrence of stem tip pain of $19 \%$. Stem tip pain causes significant disturbance to the quality of life of patients [2] and, therefore, resolution of this issue is necessary. 
A definitive cause for stem tip pain following TKA or THA is not known but suggestions have been made such as; micromotion at the bone-prosthesis interface, stress shielding resulting in bone resorption proximally at the tibial tray leading to movement at the stem, excessive stress transfer to the surrounding bone due to a mismatch in Young's modulus of elasticity between the stem and the bone and endosteal/periosteal irritation $[1,3,4,5]$. The presence of enigmatic thigh pain in THA has been reported to vary depending on the type of implant fixation, implant materials and design. An increase in the width at the distal end of a wedge shaped femoral stem from $5 \mathrm{~mm}$ to $20 \mathrm{~mm}$ was reported to result in a $40 \%$ increase in the occurrence of thigh pain [3]. In terms of the implant material a finite element study [6] identified a 30\% increase in stress levels at the anterior femoral cortical interface for cobalt chromium stem extensions in comparison to titanium stem extensions.

A literature review has identified that work is on-going to design a stem extension which will reduce the incidence of stem tip pain. However, for those patients with existing stem tip pain, avoiding another revision is critical. Therefore, a solution is needed that can modify the existing implant and/or bone in situ. One clinical case study [7] has reported attaching a cortical strut graft to the lateral aspect of the tibia to alleviate the modulus mismatch between the stem and bone in revision TKA. This study appears to provide positive results but has only reported one patient with a follow up of 1 year; therefore, the results do not provide any conclusive evidence that this is a suitable option for stem tip pain relief in TKA. The attachment of cortical strut grafts to the region of the stem tip has, however, been reported to be successful in the resolution of enigmatic thigh pain following THA [1]. The limitation in revision TKA is that there is less space available, making attachment of the graft with cables difficult. The use of a metallic bone plate may therefore simplify the surgery in avoiding the need for cabling and reducing the requirement to obtain bone allografts, with its associated risks. The senior clinician behind the current study has experienced some success in the 
management of stem tip pain, post revision TKA, with the use of metallic plates. The technique has been applied to 2 patients; 1 had complete resolution of symptoms immediately, maintained at 3 year follow-up, the other only had partial success in reducing symptoms but sufficient to allow the patient to carry on without the need for revision over a 3 year period. However, the biomechanical effect of the addition of a plate to the region of the stem tip has yet to be analysed. Therefore, the main objective of this study was to analyse the changes seen following the addition of a plate to the tibia in order to determine a possible reason for the reduction in stem tip pain seen clinically.

\section{Materials and Methods}

To achieve the objective described above, a 3D finite element model was created of the implanted tibia using the finite element software ABAQUS [8]. The model consisted of six different parts; a mobile bearing, a stem extension attached to a tibial tray, a top cement layer, a lower cement layer, a cancellous bone section and a cortical bone section as shown in Figure 1.

The connectivity of separate parts in a finite element model needs to be specified. In this study two forms of connectivity have been used, "tied surfaces" and "contact surfaces”. In the former, adjacent surfaces are fully fixed to each other whilst in the latter the surfaces can slide over each other providing the specified friction can be overcome, but they cannot penetrate each other. In the model four tied surfaces were implemented, which included the connection of the lower cement layer to the tibial cavity and the stem of the tibial prosthesis; the top cement layer was tied to the cut tibia surface on one side and to the bottom of the tibial tray on the other side. Contact surfaces between the mobile bearing and the tibial tray were modelled using the surface-to-surface contact algorithm and a coefficient of friction of 0.1 [9]. Contact between the stem extension and the cancellous bone was modelled with a surface-to-surface contact with a coefficient of friction of 0.25 [10] to represent a press-fitted 
stem extension. It should be stated that modelling full contact between the extension and the cancellous bone is an idealisation. The quality of the cancellous bone is less good distally and contact maybe patchy. It should however be noted that the modelled contact is not fully bonded but is frictional and thus can sustain relative motion if the friction is overcome. The press-fitted stem extension was chosen for modelling since this is the method considered to provide good clinical results in revision TKA in particular with regards to tibial alignment and ease of removal for further revisions [11]. In addition this was the fixation method used by the senior clinician on the stem extension where the metal plate attachment technique was applied.

The distal end of the tibia was constrained in the $\mathrm{x}, \mathrm{y}$ and $\mathrm{z}$ directions to prevent rigid body motion and a pressure load was applied to the medial and lateral surface contact patches of the mobile bearing [9], see Figure 2. The model was based upon the leg in late stance phase ( $40 \%$ of gait cycle) where the joint reaction force is maximum and the majority of loading is reported [12] to be on the medial condyle. Morrison [12] used experimental data from 14 normal adults to calculate, through the use of biomechanical principles, the location of the centre of pressure at this point of the gait cycle. On this basis the distribution of loading on the medial and lateral condyles for this model was calculated to be approximately $70 \%$ and $30 \%$ respectively. A compression force of $2208 \mathrm{~N}$ was applied. This was based on a pressure load of three times the body weight of a $75 \mathrm{~kg}$ person due to the combined effect of body weight and the gastrocnemius muscle loading in late stance phase [12].

A rectangular plate (90 mm x $15 \mathrm{~mm}$ x $5 \mathrm{~mm}$ ) was used for the initial studies, based on a standard 4-hole locking compression plate (LCP, Synthes Ltd, Welwyn Garden City, UK) similar to that used by the senior clinician. The positioning of the plate in the finite element model was such that it was placed as close to the surface of the tibia as possible in the dysphyseal area at the stem tip. It was equidistant on the medial and lateral side of the stem 
tip in the superior/inferior directions, as shown in Figure 3 (b) and (c). The full surface of the plate was constrained using a tied surface to the tibia to model a fully integrated bone plate condition.

Further detailed analysis was also completed by refining the plate attachment method and conducting an analysis of different plate material options. The refined attachment method of the plate to the tibia applied fixation through only four tied surfaces of $5 \mathrm{~mm}$ diameter, rather than the full surface area. This simulated the initial screwed attachment of the plate to the tibia with 4 uni-cortical screws. The most commonly used bone plate materials in fracture repairs are titanium and stainless steel; therefore, these materials have been used in this study. In addition, plates of cortical bone were also modelled as they have been reported to be used in revision THA with some success as a possible solution to stem tip pain [1].

The implanted tibial model was meshed using quadratic solid tetrahedral elements, the plate was meshed using quadratic brick elements for the full surface tied plate and quadratic solid tetrahedral elements for the models with the refined plate attachment method.

Material properties were applied and all parts were assumed to be linearly elastic, homogenous and isotropic [11]. The material properties used for the various parts of the models are shown in Table 1.

\section{Results}

Figure 3 shows the von Mises stress plots for the un-plated and medially and laterally attached, plated (titanium) models. The plots show the anterior of the tibia sliced through the coronal plane to demonstrate the area of stress at the stem tip. A threshold value of $1 \mathrm{MPa}$ was arbitrarily chosen for the von Mises stress plots since it showed the concentrations of stress at the stem tip most clearly. 
When the plate is attached to the tibia the area of von Mises stress concentration above the threshold level at the stem tip is reduced in comparison to the un-plated model. This demonstrates that the addition of the plate has an impact on the von Mises stress levels at this location. Furthermore, the addition of the plate affects the stress levels in the bone immediately under the plate. Increases in von Mises stress levels are present in the bone at the edges of the plate (where load transfer occurs) in comparison to the un-plated model, whilst underneath the plate there is a reduction in von Mises stress levels.

Examining the effect of the location of the plate on the medial or lateral aspect of the tibia it is noted that attaching the plate to the medial side of the tibia results in the smallest area of von Mises stress concentration above the threshold value. This indicates that for this particular finite element model the medial aspect of the tibia may be the preferred location for the plate. This is examined in further detail in the discussion section.

Figure 4 shows a similar plot to Figure 3 but for the tibia with no stem extension. This serves as a reference or comparison configuration where stem tip pain is not a problem. The same contour levels have been used to facilitate comparison with Fig 3. It can be seen that the zone of material above the threshold is smaller than in any configuration with a stem extension. A further insight is that the cancellous stress in the proximal region are slightly higher, this is because there is no stem extension to transfer the load distally.

Figure 5 shows the results of the model with the initially screwed plate. Comparing Figure 3 and Figure 5 it can be seen that the initially screwed plate results in a similar reduction of area of von Mises stress concentrations at the stem tip as the fully integrated plate. However, the initially screwed plate has a larger impact on the von Mises stress levels in the bone immediately surrounding the plate. There is a greater increase in von Mises stress concentrations in the bone at the ends of the plate but the loading in the bone under the plate surface has not been reduced to the same extent as for the fully integrated plate. 
When the plate is initially attached, contact is likely to be similar to the screwed plate model. However, over time, it is believed that the bone remodels and the bone growth results in the plate becoming fully bonded to the tibia. Therefore, once bone remodelling has taken place the contact between the plate and the tibia in-vivo is considered to be better represented by the fully integrated plate model.

The models were also completed with the three different plate materials (cortical bone, titanium, and stainless steel) to determine what effect the different materials would have on stress levels seen at the stem tip. The von Mises stress plots of these models are shown in Figure 6. As the stiffness of the plate is increased the area of von Mises stresses above the threshold level at the stem tip reduces slightly. What is more noticeable is that the loading in the bone immediately surrounding the plate changes as the plate increases in stiffness. In particular the loading in the bone under the surface of the plate is reduced as the stiffness of the plate is increased.

In order to further quantify the bone stresses, charts showing the relative volume of cancellous bone with a stress above $1 \mathrm{MPa}$ have been produced. The baseline for these data is the volume of cancellous bone meeting this criterion in the un-plated model. A full discussion of these charts is given in the following section, but in essence a lower relative volume could be considered to indicate a more benign configuration. Such charts have been produced comparing the position of the plate (Figure 7) and the plate material (Figure 8). Finally, to consider the integrity of the plate material the maximum plate von Mises stresses have been extracted and are presented in Figure 9.

\section{Discussion}

The results have confirmed that the addition of a plate to the tibia reduces the stress concentrations in the bone at the stem tip of the implant. However, the addition of a plate also 
has an impact on the loading in the bone surrounding the plate and this is dependent on the plate material and the surface attachment method.

In order to quantify any changes occurring and to compare the different models in further detail the volume of cancellous bone at the stem tip above the 1MPa threshold level was calculated. For the purposes of this study this was considered to be the volume of 'critically stressed bone'. As mentioned previously the threshold value of $1 \mathrm{MPa}$ was selected as an arbitrary value to characterise the area of stress at the stem tip. The cancellous bone was chosen for analysis because the areas of higher stress were located in the cancellous bone at the stem tip and could be isolated more easily from the rest of the model. The un-plated model was assumed to be the worst-case scenario (100\%) and therefore taken as a reference point from which to calculate the percentage difference in critically stressed bone in the other models. The percentage difference in volume of critically stressed bone between the unplated stem extended and the standard stem model (stress plots not shown in this paper) with no stem extension was also calculated. Enigmatic stem tip pain is reported to be associated with stem extended implants and not with standard stems; therefore, the standard stem model has been used to compare the plated models with what would nominally be considered to be 'pain-free’ stress levels.

The results of these calculations are shown in Figure 7 and this shows that the addition of a titanium plate to the tibia reduces the volume of critically stressed bone at the stem tip in comparison with the un-plated model. The largest reduction is when the plate is on the medial side, which is the side under the greatest compressive stress, due to the increased loading on the medial condyle. The addition of the plate moves the neutral axis towards the side of the tibia to which it is attached, and increases the cross sectional area, providing a reduction of the axial stresses in this region. To have the greatest impact on the stresses in the tibia the plate must be positioned on the side carrying greatest loading, allowing the plate to be used to 
maximum benefit. Positioning of the plate on the opposite side will have an effect on the stresses on that side but this will not counteract the impact of the higher loading on the other side, as demonstrated in Figure 3. The results have shown that the optimum location for the plate for this particular finite element model is the medial side, since this is where the highest stresses occur. This may vary clinically according to bone morphology and stem alignment. It is noted that the senior clinician currently attaches the plate on the anterior-medial aspect of the tibia for ease of placement.

The percentage reduction in the volume of critically stressed cancellous bone at the stem tipin the model with the plate on the medial side of the tibia approaches the volume of critically stressed bone in the standard stem model (where stem tip pain is not considered an issue), see Figure 7. Therefore if stem tip pain is caused by high stress concentrations at the stem tip, these results suggest that the attachment of a plate is the reason for the reduction in stem tip pain seen clinically. However, a definitive cause of stem tip pain remains unknown.

In the initially screwed plate (Figure 5), a change to the loading in the bone may be anticipated since the load is being transferred through smaller, more localised, regions rather than through the full surface, as in the fully integrated plate. This could potentially provide a risk to the bone at the edges of the plate where increased stress levels are present. However, due to the mechanism of continuous bone resorption and formation, known as 'Wolff's law' [13], the plate is expected to become more integrated with the tibia over time resulting in a situation approaching that represented by the fully integrated plate model where the stress levels at the ends of the plate are lower. This is likely to be more true for the titanium than the stainless steel plate. The long-term effect of the change in loading on the strength and function of the tibia is not known and is an area which requires further investigation.

The results of the variation in the volume of critically stressed cancellous bone at the stem tip with different plate materials are shown in Figure 8. This graph highlights that the increased 
stiffness of the plate materials results in a reduction in the volume of critically stressed cancellous bone at the stem tip. The difference between the stainless steel plated model and the cortical bone plated model is $\sim 7 \%$, indicating that there may be a potential advantage in using a stiffer plate material.

Examining the maximum von Mises stresses in the different plate materials, it can be seen that these correspond with the higher volume of critically stressed cancellous bone at the distal end of the plate. The highest von Mises stress levels are in the stainless steel plate, as shown in Figure 9. When the load is applied to the mobile bearing it is transferred through the stem extension, into the bone and to the plate. As the stainless steel plate is stiffer than the other materials the load transfer from the bone into the plate is greater. This results in a higher load in the plate and hence higher stresses in the plate. However, it also results in a higher stress concentration at the end of the plate as this load has to pass back into the bone. Calculation of the volume of critically stressed cancellous bone at the distal end of the stainless steel plate was approximately $44 \%$ of the volume of critically stressed bone at the stem tip in the un-plated model. This confirms that the stiffer plate introduces a relatively significant level of loading at this location. This mechanism of load transfer also reduces the loading present in the bone located underneath the plate surface. This could lead to loosening of the plate or reduced strength in the bone surrounding the stem extension. The greatest change in loading was seen in the stainless steel plate; however, this could be a potential risk with all plate materials depending on attachment method, as discussed previously.

On this basis, the choice of material for the plate is considered to be a compromise between reductions in von Mises stress concentrations at the stem tip whilst avoiding a significant alteration to the loading in the bone surrounding the plate. From the results of the models presented here, the optimum option would be to use a titanium plate as it has a high reduction in the volume of critically stressed cancellous bone at the stem tip for a lower level of change 
to the loading elsewhere in the bone. Titanium would also be the preferred option in comparison to cortical bone since it avoids issues with obtaining allografts and the risks associated in using them. However, further investigations are required both with additional modelling and clinical data to determine what the long term implications of the different materials are.

This study has investigated the clinical technique of applying a plate to the tibia at the location of the stem extension of an implant to reduce the effects of stem tip pain. From the analysis conducted it has been concluded that the attachment of a plate to the tibia has an impact on von Mises stress levels in the cancellous bone at the stem tip. In all the models, the plate reduced the volume of critically stressed cancellous bone at the stem tip, but the amount of reduction was dependant on stem extension type, plate attachment method, plate location and material used. The stresses were reduced to around the levels found in the standard stem model, which is not reported to have the issue of stem tip pain. Therefore the results show evidence that this method could be a viable option for the reduction of enigmatic stem tip pain.

Since the addition of the plate has been seen to result in a reduction of pain clinically, it is hypothesised that stem tip pain may indeed be caused as a consequence of increased loading and stress transfer to the bone at the tip of the stem extension. This is in agreement with other studies completed on the possible origins of enigmatic stem tip pain in THA or TKA [1, 3, 4, 5]. Whilst the clinical data of the effects of the addition of a plate to the stem tip region have yet to be reported, our experience from a small sample set is that patients can experience both early and sustained pain relief from this relatively simple procedure, as discussed in Section 2.

There are a number of limitations with the models used such as the simplification of bone material properties; however, for the purposes of this study exact values were not required 
from the finite element models as the objective was to identify relative changes in stress values seen at the stem tip. Therefore, the conclusions provided here are not considered to have been affected by the limitations of the model.

\section{References}

1 Brown, T.E., Larson, B., Shen, F. \& Moskal, J.T: Thigh Pain After Cementless Total Hip Arthroplasty: Evaluation and Management. J Am Acad Orthop Surg, 2002;10(6):385

2 Barrack, R.L., Rorabeck, C., Burt, M. \& Sawhney, J: 1999, Pain at the end of the stern after revision total knee Arthroplasty. Clin Orthop Relat Res 1999;367:216

3 Vresilovic, E.J., Hozack, W.J. \& Rothman, R.H: Incidence of thigh pain after uncemented total hip arthroplasty as a function of femoral stem size. J Arthroplasty 1996;11(3):304

4 Lavernia, C., D’Apuzzo, M., Hernandez, V. \& Lee, D: Thigh pain in primary total hip arthroplasty: The effects of elastic moduli. J Arthroplasty 2004;19(7)(suppl 2):10

5 Huiskes, H: The various stress patterns of press-fit, ingrown, and cemented femoral stems. Clin Orthop Relat Res 1990;261:27

6 Namba, R.S., Keyak, J.H., Kim, A.S., Vu, L.P. \& Skinner, H.B: Cementless implant composition and femoral stress: A finite element analysis. Clin Orthop Relat Res $1998 ; 347: 261$

7 Glenn, J.C., Sokoloski, S.N., Damer, B.M. \& Tabit, J.M: Tibia pain at end of stem with stemmed revision total knee arthroplasty: treatment with cortical strut graft technique. J Arthroplasty 2010;25(3):497.e1 
8 Frehill, B: Initial and Long Term Stability of Augmented Knee Arthroplasty using Finite Element Methods. PhD Thesis 2010

9 Villa, T., Migliavacca, F., Gastaldi, D., Colombo, M. \& Pietrabissa, R: Contact stresses and fatigue life in a knee prosthesis: comparison between in vitro measurements and computational simulations. J Biomech 2004;37(1):45

10 Kim, Y.H., Kwon, O.S. \& Kim, K: Analysis of biomechanical effect of stem-end design in revision TKA using Digital Korean model. Clin Biomech (Bristol, Avon) 2008;23(7):853

11 Completo, A., Talaia, P., Fonseca, F. \& Simões, J.A: Relationship of design features of stemmed tibial knee prosthesis with stress shielding and end-of-stem pain. Mater Des 2009;30(4):1391

12 Morrison, J.B: The mechanics of the knee joint in relation to normal walking. $\mathrm{J}$ Biomech 1970;3(1):51

13 Wolff, J: Das Gesetz der Transformation der Knochen. Berlin: Hirschwald. 1892

14 Nyman, J.S., Hazelwood, S.J., Rodrigo, J.J., Martin, R.B. \& Yeh, O.C: Long stemmed total knee arthroplasty with interlocking screws: a computational bone adaptation study. J Orthop Res 2004;22(1):51 


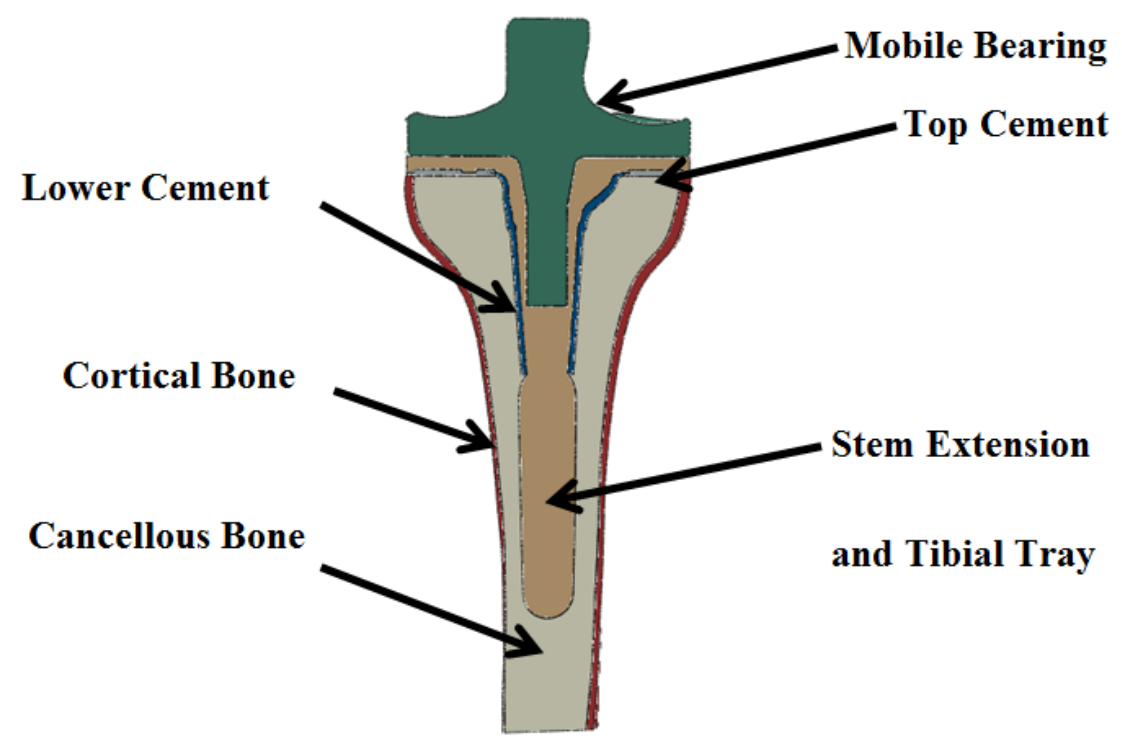

Figure 1: Stem extension model

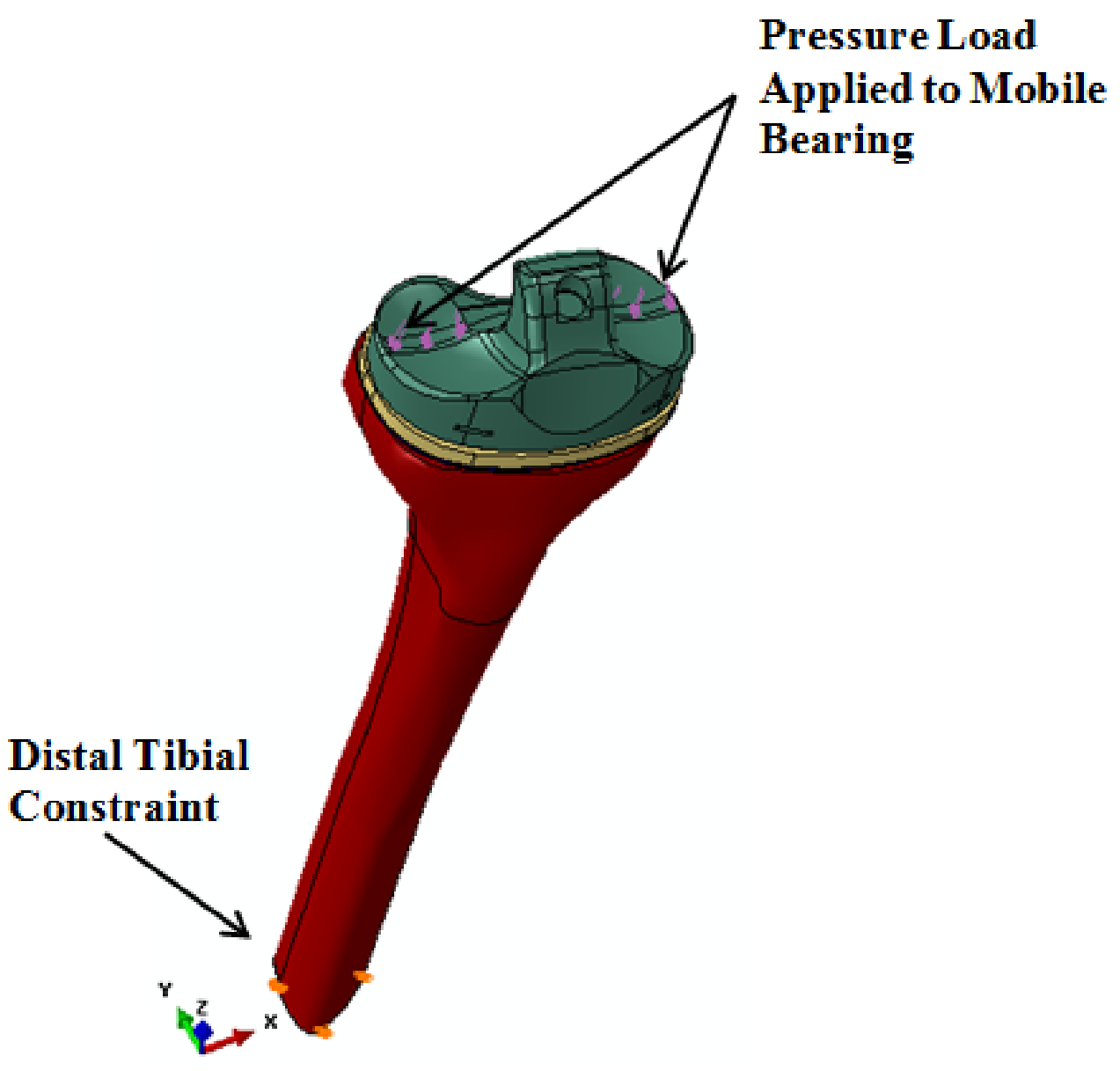

Figure 2: Completed 3D Finite Element model showing tibial constraint and bearing loading 

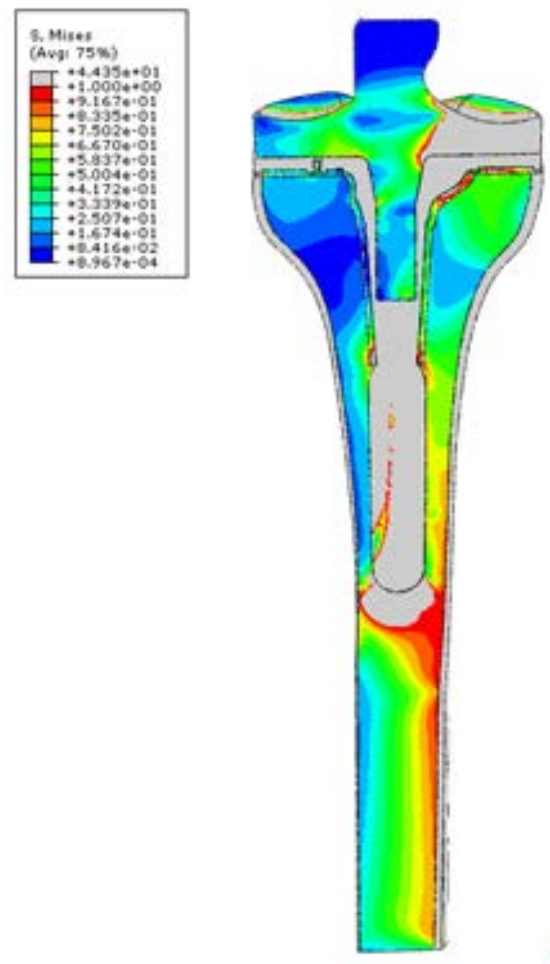

(a)
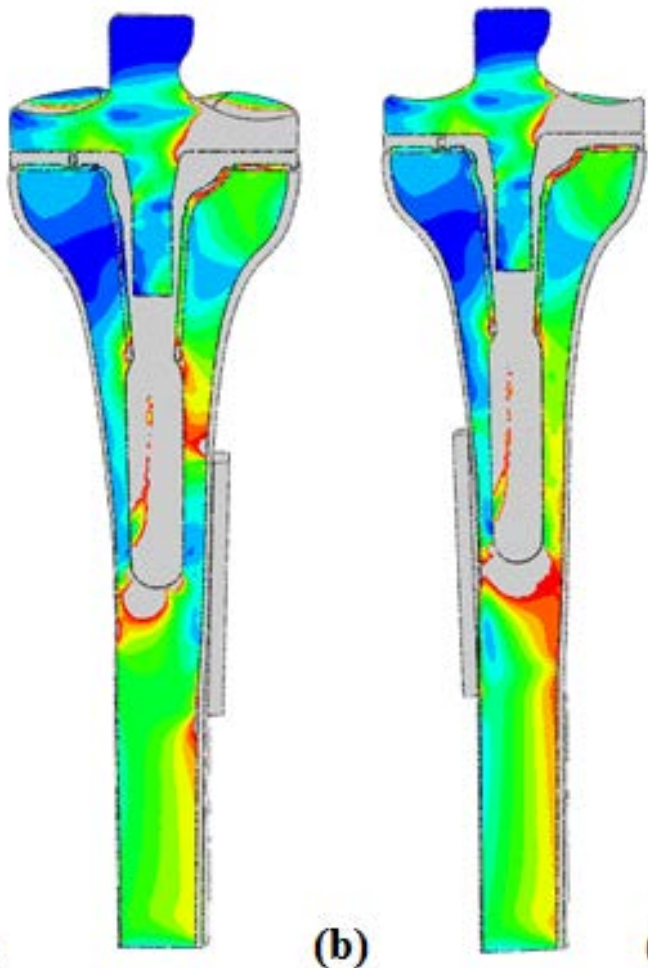

(c)

Figure 3: von Mises stress plots showing (a) un-plated, (b) medially plated and (c) laterally plated models (titanium plates)
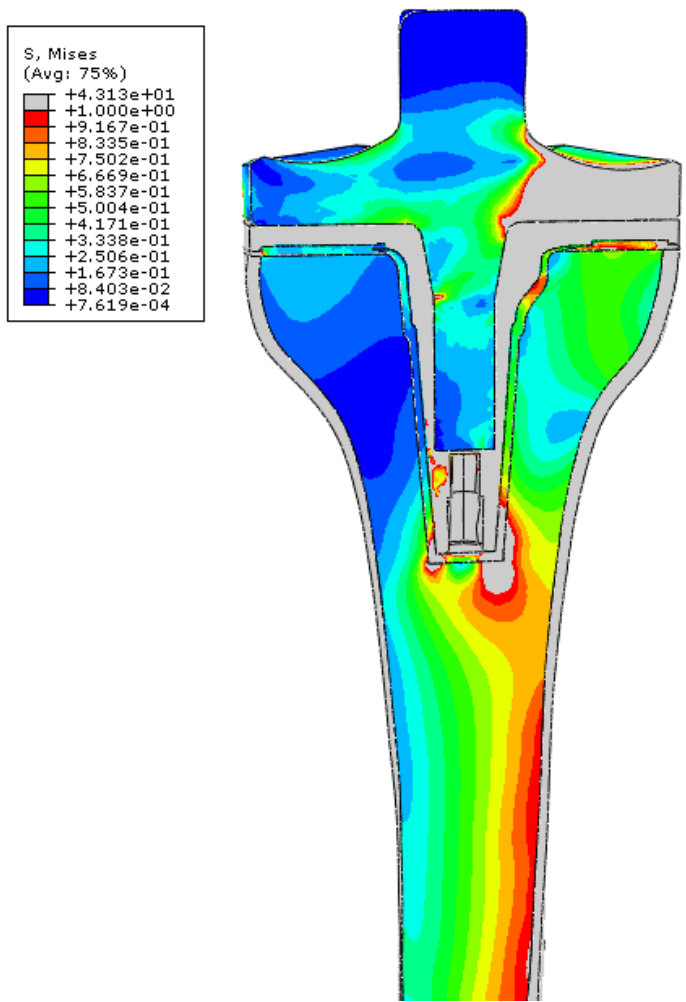

Figure 4: von Mises stress plot for the tibia with no stem extension 


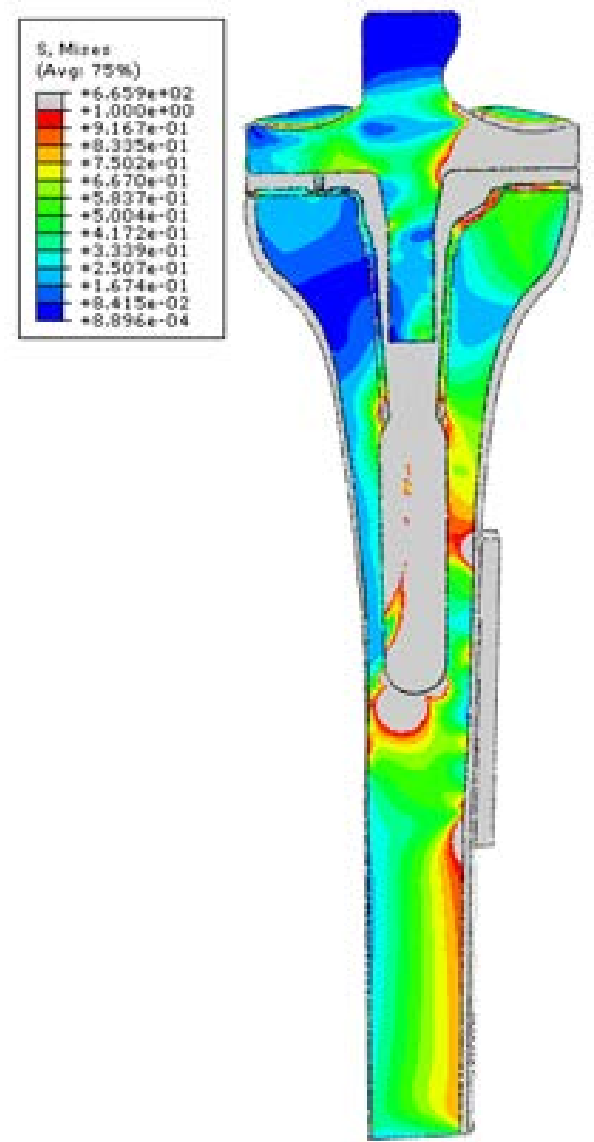

Figure 5: von Mises stress plot showing the initially screwed titanium plate model
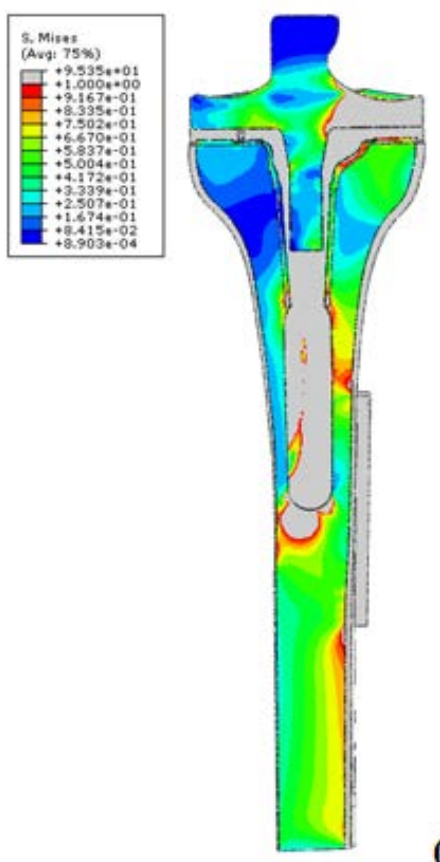

(a)

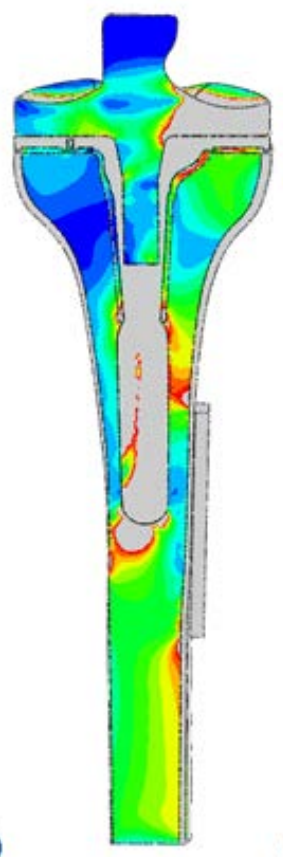

(b)

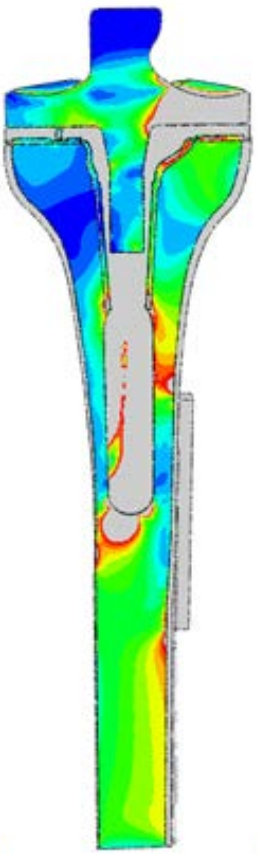

(c)

Figure 6: von Mises stress plots showing (a) cortical, (b) titanium and (c) stainless steel plated models 


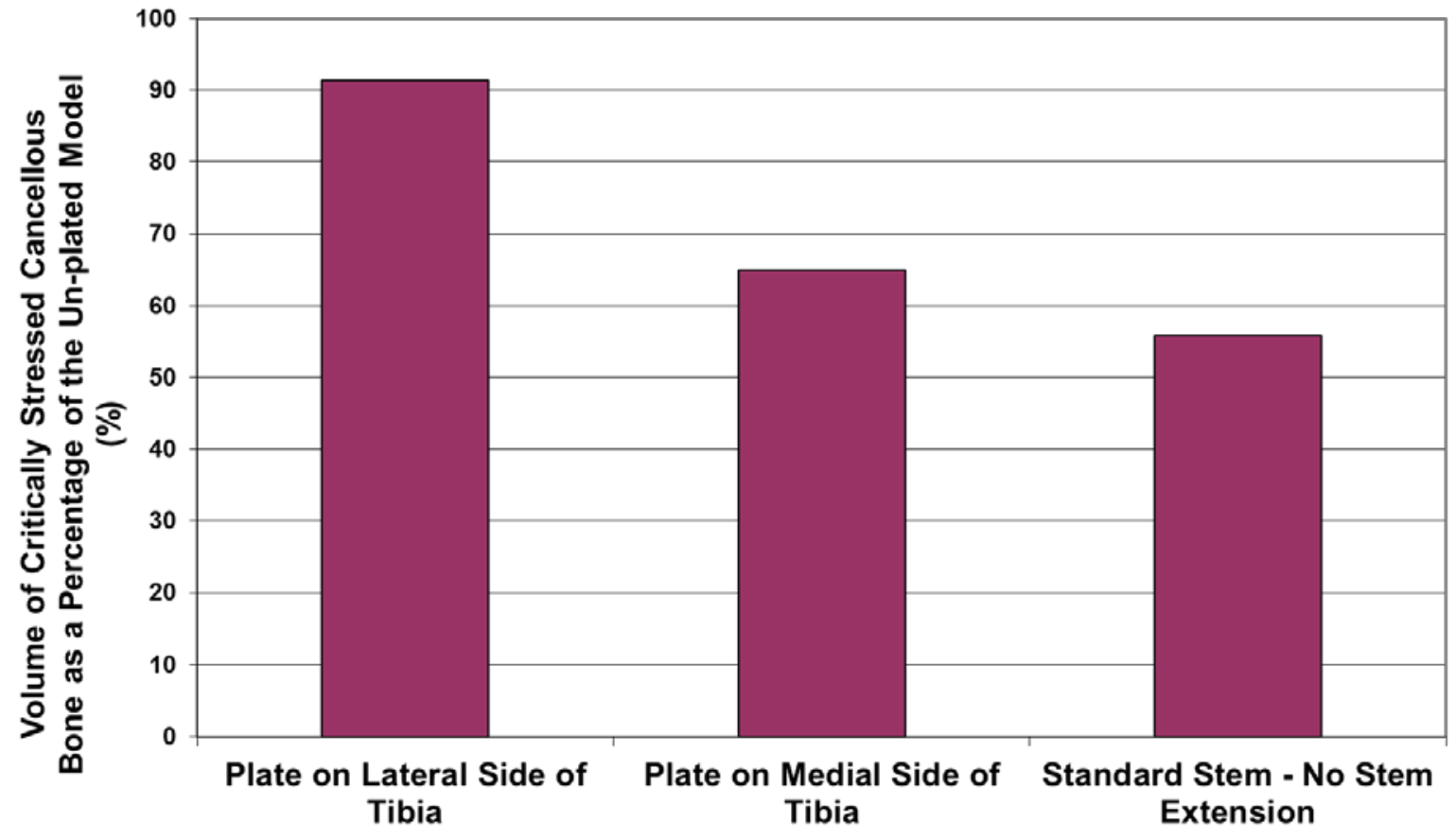

Figure 7: Comparison of critically stressed cancellous bone at stem tip as a percentage of un-plated model

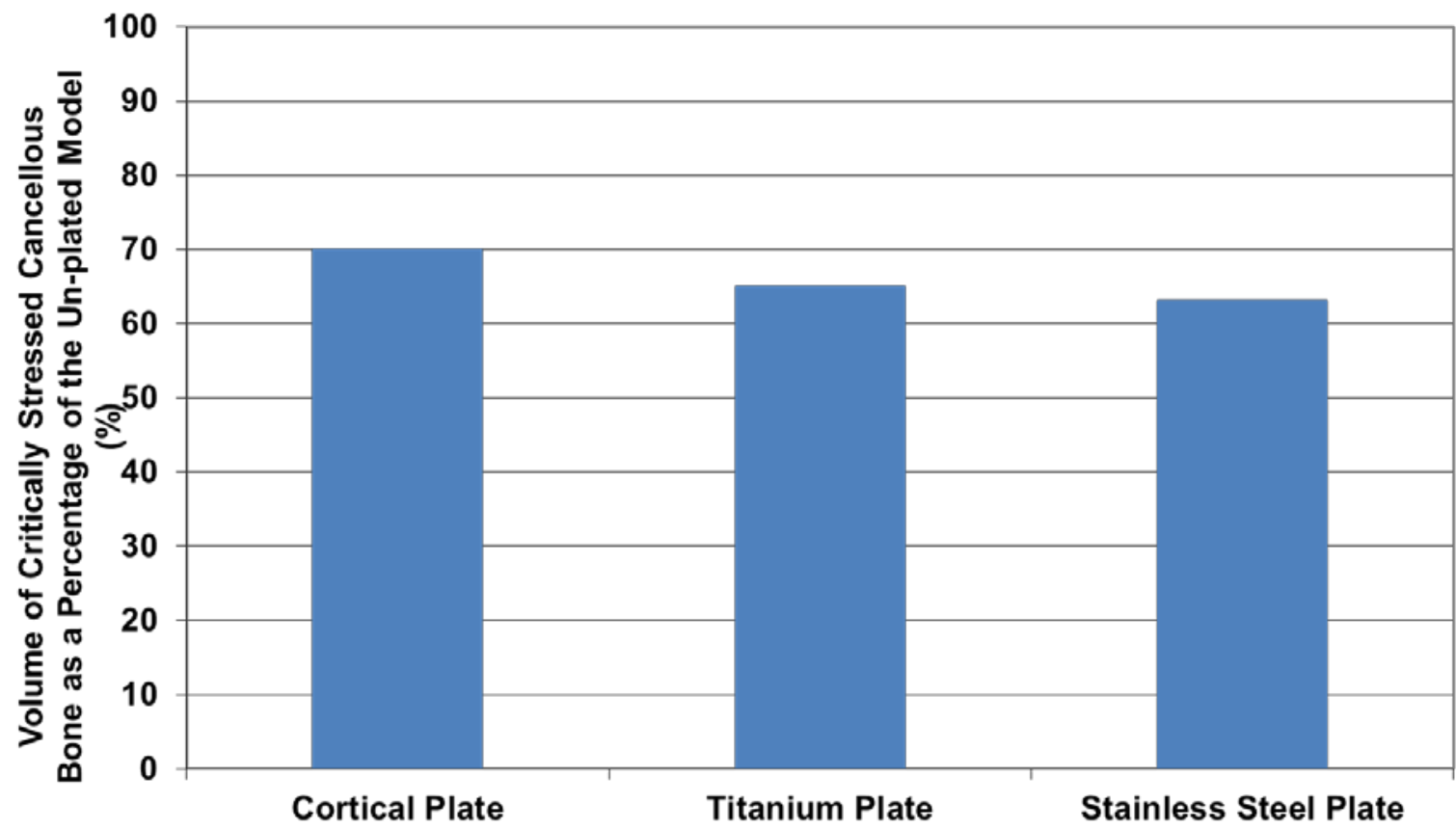

Figure 8: Comparison of critically stressed cancellous bone at stem tip as a percentage of the un-plated model 


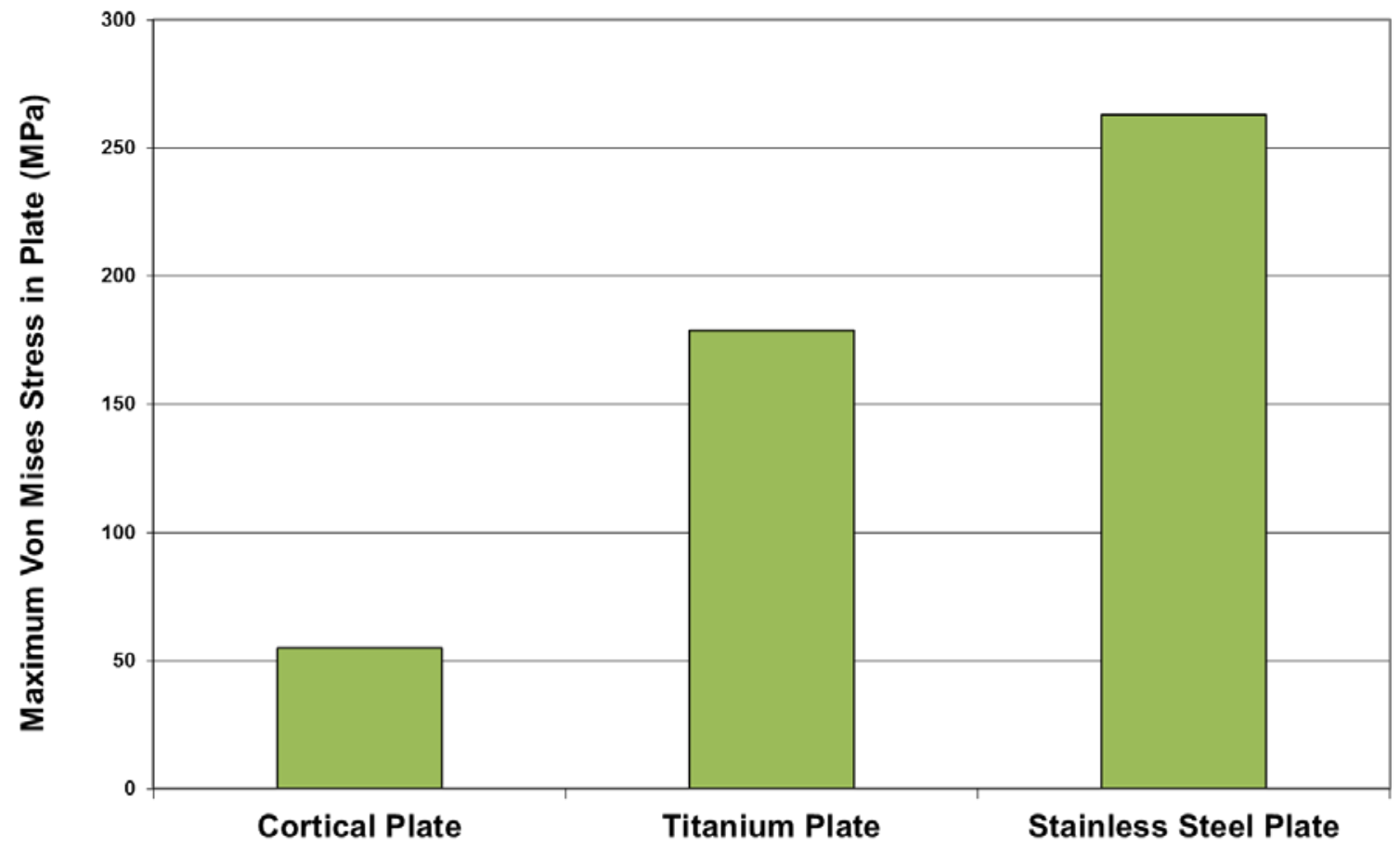

Figure 9: Maximum von Mises stress in the plate for the different plate materials

\begin{tabular}{ccc}
\hline Material & Young's Modulus of & Poisson's Ratio \\
& Elasticity E (GPa) & v \\
\hline Cancellous Bone & 0.7 & 0.30 \\
\hline Cortical Bone & 17 & 0.30 \\
\hline PMMA (Cement) & & 0.46 \\
\hline Ti6Al4V (Standard and press-fit stems) & 110 & 0.30 \\
\hline UHMWPE (Bearing) & & 0.25 \\
\hline Stainless Steel (316) & 2.3 & 0.3 \\
\hline
\end{tabular}

Table 1: Material properties used in models [5, 14] 
